\title{
Synthesis and Biological Activity of New Donepezil-Hydrazinonicotinamide Hybrids
}

\author{
Authors \\ Affiliation \\ Key words \\ inhibitors of acetylcho- \\ linesterase \\ - Alzheimer's disease \\ donepezil
}

\author{
E. Żurek, P. Szymański, E. Mikiciuk-Olasik
}

Department of Pharmaceutical Chemistry and Drug Analyses, Medical University, Lodz, Poland

\begin{abstract}
$\nabla$

Currently available treatment used in Alzheimer's disease is based on acetylcholinesterase inhibitors, e.g. donepezil, tacrine, galantamine, and rivastigmine. In the present study some derivatives of donepezil were synthesized, and their potential anticholinesterase properties were investigated using the colorimetric Ellman's method. These compounds were syn-
\end{abstract}

\section{Introduction}

$\nabla$

Alzheimer's disease (AD) is a slow progressive, degenerative disorder of the CNS. It is the most common form of dementia accounting for about $50-60 \%$ of all cases of dementia among persons over 65 years of age. Currently, an estimated 4.5 million older people suffer with Alzheimer's disease (AD), and researchers predict that by 2050 the number could nearly triple, to 13.5 million [1].

This disease is important not only because of the number of affected patients but also because it leads to significant physical and emotional burden on families and caregivers.

Alzheimer's disease is characterized by the loss of memory and learning ability, together with a reduced ability to perform basic activities of daily living. AD patients exhibit marked neuropsychiatric symptoms such as apathy, irritability, anxiety, depression, hallucinations and verbal and physical agitation [2].

Studies performed during the last 20 years revealed that disordered cholinergic transmission is behind cognitive impairment present in Alzheimer's disease patients. The disorders in transmission result from the reduced number of cholinergic neurons in brain regions associated with higher cognitive functions, i.e. in the neocortex and hippocampus, as well as from the thesized by condensation between indanone derivatives and the hydrazine nicotinated moiety (Hynic). For received derivatives, the selectivity and the $\mathrm{IC}_{50}$ values for acetylcholinesterase and butyrylcholinesterase were calculated. All the tested compounds exhibited lower affinity for AChE than donepezil and higher affinity for $\mathrm{BChE}$ than donepezil. Compound $\mathbf{3 3}$ showed the most selectivity for AChE among the obtained indanone derivatives.

decreased level of choline acetyltransferase, leading to impaired synthesis and uptake of acetylcholine (ACh) neurotransmitter $[3,4]$. As ACh is degraded by cholinesterase, it stands to reason that cholinergic transmission can be improved by inhibiting the activity of this enzyme.

2 kinds of choline esterases catalyzing hydrolysis of choline esters exist in the central nervous system: acetylcholinesterase (AChE), the so-called true esterase, and butyrylcholinesterase (BChE), referred to as pseudocholinesterase or non-specific cholinesterase. AChE is bound to the membrane of cholinergic neurons, whereas $\mathrm{BChE}$ is present both in the neurons and in the glial cells. Both enzymes show 65\% homology, and the main differences pertain to their substrate specificity.

Acetylcholinesterase selectively and rapidly hydrolyzes acetylcholine in cholinergic synapses. In turn, butyrylcholinesterase can also hydrolyze butyrylcholine and some medications and drugs, aside from acetylcholine. Moreover, it regulates cholinergic transmission in states of acetylcholinesterase deficiency. In healthy individuals, AChE accounts for $80 \%$ of esterase activity within the central nervous system, whereas the remaining $20 \%$ is provided by BChE. In Alzheimer's disease, the activity of AChE can be reduced to $67 \%$ of normal level in certain brain regions; simultaneously, an 
increase in BChE level is observed, reaching up to $165 \%$ of its normal level $[5,6]$.

Cholinesterase inhibitors (ChEI) constitute an important group of compounds used in the symptomatic treatment of Alzheimer's disease. They support cholinergic transmission by inhibiting acetylcholinesterase cleavage. Most commonly administered agents from this group include donepezil and galantamine, both being acetylcholinesterase inhibitors (AChEI), and rivastigmine, which inhibits both acetylcholinesterase and butyrylcholinesterase (AChEI, BChEI). Studies revealed that the administration of such double inhibitors is particularly favorable at later stages of Alzheimer's disease [7-10].

In the study, synthesis and biological evaluation of a series of new donepezil derivatives with hydrazine nicotinate moiety as potential cholinesterase inhibitors are described.

Inhibitory activity of acetylcholinesterase and butyrylcholinesterase of the obtained compounds was studied using the method of Ellman, to determine the rate of hydrolysis of acetyltiocholine (ATCh) in the presence the inhibitor [11].

\section{Material and Methods}

$\nabla$

\section{Chemistry}

Reaction was monitored by TLC using DC-Alufolien Kieselgel 60F254 plates (Merck), with detection by UV lamp $(254 \mathrm{~nm})$. Melting points were measured on an Electrothermal apparatus in open capillaries and are uncorrected. Column chromatography was performed using silica gel 60 (230-400 mesh, Merc). IR spectra were recorded in $\mathrm{KBr}$ using a Mattson Infinity Series FT-IR spectrophotometer. ${ }^{1} \mathrm{H}$ NMR spectra were recorded with a Varian Mercury $300 \mathrm{MHz}$ spectrometer, using tetramethylsilane as internal standard. Elemental analyses were recorded using Perkin Elmer series II, CHNSO, Analyzer 2400.

Mass spectra were performed by the Centre of Molecular and Macromolecular Studies in Lodz.

\section{6-hydrazinopyridine-3-carboxylic acid (1)}

6-chloronicotinic acid ( $50.8 \mathrm{mmol}$ ) was added to $80 \%$ hydrazine hydrate $(930.0 \mathrm{mmol})$ and placed in a $100^{\circ} \mathrm{C}$ oil bath for $4 \mathrm{~h}$. The homogenous reaction mixture was cooled to room temperature and concentrated to dryness to give a white solid. The solid was dissolved in water and on acidification to $\mathrm{pH} 5.5$ with concentrated hydrochloric acid, a precipitate was formed. The precipitate was isolated by filtration and was washed with ethanol and ether, and dried in vacuum.

Yield $85 \%$. Mp. $295-297^{\circ} \mathrm{C}$. IR (KBr) cm $\mathrm{cm}^{-1} 1690,3229,3308 .{ }^{1} \mathrm{H}$ NMR (DMSO) $\delta: 8.5(1 \mathrm{H}, \mathrm{s}, \mathrm{COOH}), 8.2(1 \mathrm{H}, \mathrm{s}, \mathrm{CHN}), 7.8(1 \mathrm{H}, \mathrm{d}$, CCHC), 6.7 (1H, d, CCHC), $3.3(1 \mathrm{H}, \mathrm{s}, \mathrm{CNH}), 2.5\left(2 \mathrm{H}, \mathrm{d}, \mathrm{NH}_{2}\right)$. Anal. calc. for $\mathrm{C}_{6} \mathrm{H}_{7} \mathrm{~N}_{3} \mathrm{O}_{2}$ (\%): C 47.06, $\mathrm{H}$ 4.61, N 27.44; Found (\%): C 46.78, H 4.35, N 27.11.

\section{6-Boc-hydrazinopyridine-3-carboxylic acid (2)}

To a solution $1(9.8 \mathrm{mmol})$ and triethyl amine $(11.8 \mathrm{mmol})$ in DMF $(10 \mathrm{~mL})$ was added di-tert-butyl dicarbonate $(9.8 \mathrm{mmol})$. The reaction mixture became homogenous after $1 \mathrm{~h}$ and stirring was continued for $16 \mathrm{~h}$ at room temperature. The reaction mixture was concentrated to dryness under reduced pressure to give brown solid. Recrystallization from ethyl acetate gave product $\mathbf{2}$ as a white solid.

Yield 75\%. Mp. $285-287^{\circ} \mathrm{C}$. IR (KBr) cm ${ }^{-1}: 1611,1706,3248 .{ }^{1} \mathrm{H}$

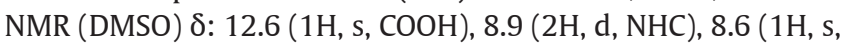

CCHC), 8.0 (1H, d, CCHC), 6.5 (1H, d, CCHC), 3.3 (1H, d, CNH), 1.4 (9H, s, BOC). Anal. calc. for $\mathrm{C}_{11} \mathrm{H}_{15} \mathrm{~N}_{3} \mathrm{O}_{4}$ (\%): C 52.17, $\mathrm{H} \mathrm{5.93,} \mathrm{N}$ 16.59; Found (\%): C 52.08, H 5.73, N 16.67.

\section{General procedure for the synthesis of compounds (5)} and (6)

5-hydroxyindan-1-one ( $5.0 \mathrm{mmol})$, paraformaldehyd $(5.0 \mathrm{mmol})$ and the corresponding amine were dissolved in isopropanol $(20 \mathrm{~mL})$. Afterwards, the $\mathrm{pH}$ of the suspension was adjusted to 2-3 with concentrated hydrochloric acid. The mixture was refluxed for $5 \mathrm{~h}$. The solution was them evaporated to the half of the initial volume and crude precipitate was filtered off. The dry solid was recrystallized from methanol.

\section{5-hydroxy-2-piperidin-1-ylmethylindan-1-one}

hydrochloride (5)

Yield $78 \%$. Mp. $165-167^{\circ} \mathrm{C}$. IR (KBr) $\mathrm{cm}^{-1}: 1593,1697,2737$, 2498, 3020. ${ }^{1} \mathrm{H}$ NMR (DMSO) $\delta: 10.4$ (1H, b, OH), 7.5 (1H, m, Ar), 6.7-6.8 (2H, m, Ar), 3.5 (2H, d, $\mathrm{CHCH}_{2}$ ), 3.4 (d, 2H, $\mathrm{NCH}_{2}$ ), 3.0-3.1 (4H, m, NCH $), 2.7-2.8(1 \mathrm{H}, \mathrm{m}, \mathrm{COCH}), 1.5-1.7\left(6 \mathrm{H}, \mathrm{m}, 3 \cdot \mathrm{CH}_{2}\right)$. MS (FAB) (m/z): $246(\mathrm{M}+1)$. Anal. calc. for $\mathrm{C}_{15} \mathrm{H}_{20} \mathrm{ClNO}_{2}$ (\%): C 63.94, H 7.17, N 4.98; Found (\%): C 63.69, H 7.09, N 4.87 .

\section{2-(4-benzylpiperidin-1-ylmethyl)-5-hydroxyindan-1-one hydrochloride (6)}

Yield $68 \%$. Mp. $170-172{ }^{\circ} \mathrm{C}$. IR (KBr) $\mathrm{cm}^{-1}: 739,1600,1695$,

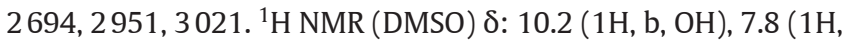
$\mathrm{m}, \mathrm{Ar}), 7.2-7.4(5 \mathrm{H}, \mathrm{m}, \mathrm{Ar}), 6.8-6.9(2 \mathrm{H}, \mathrm{m}, \mathrm{Ar}), 3.6-3.7(1 \mathrm{H}, \mathrm{m}$, $\mathrm{COCH}), 3.4\left(2 \mathrm{H}, \mathrm{d}, \mathrm{NCH}_{2}\right), 3.0\left(2 \mathrm{H}, \mathrm{d}, \mathrm{CHCH}_{2}\right), 2.7\left(2 \mathrm{H}, \mathrm{t}, \mathrm{ArCH}_{2}\right)$, 2.5-2.6 (4H, m, $\left.\mathrm{NCH}_{2}\right), 1.3-1.6\left(5 \mathrm{H}, \mathrm{m}, \mathrm{CH}_{2} \mathrm{CHCH}_{2}\right)$. MS (FAB) $(\mathrm{m} / \mathrm{z}): 336(\mathrm{M}+1)$. Anal. calc. for $\mathrm{C}_{22} \mathrm{H}_{26} \mathrm{ClNO}_{2}(\%)$ : $\mathrm{C} 71.05, \mathrm{H}$ 7.05, N 3.77; Found (\%): C 69.78, H 6.91, N 3.53.

General procedure for synthesis of compounds (7) and (8) To solution of $1 \mathrm{M} \mathrm{NaOH}(20.1 \mathrm{mmol})$ in tert-butyl alcohol $(6 \mathrm{~mL})$ was added the corresponding n-bromoalkylamine hydrobromide $(9.1 \mathrm{mmol})$ and di-tert-butyl dicarbonate $(10.1 \mathrm{mmol})$. The reaction mixture was stirred for $12 \mathrm{~h}$ in room temperature, and then was washed with $0.1 \mathrm{M} \mathrm{HCl}$, and $5 \% \mathrm{NaHCO}_{3}$, and brine. The organic layer was dried over $\mathrm{Na}_{2} \mathrm{SO}_{4}$ and concentrated in vacuo to give N-Boc-3-bromoalkylamine as yellow oil.

\section{N-Boc-2-bromoethylamine (7)}

Yield $84 \%$. IR (KBr) cm ${ }^{-1}:$ 1692, 2968, 3280. ${ }^{1} \mathrm{H}$ NMR (DMSO)

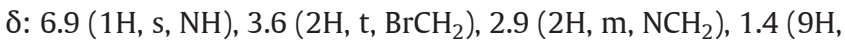
$\mathrm{s}, \mathrm{Boc})$.

\section{N-Boc-3-bromopropylamine (8)}

Yield $87 \%$. IR (KBr) cm ${ }^{-1}: 1690,2972,3285 .{ }^{1} \mathrm{H}$ NMR (DMSO) $\delta$ : $6.9(1 \mathrm{H}, \mathrm{s}, \mathrm{NH}), 3.5\left(2 \mathrm{H}, \mathrm{t}, \mathrm{BrCH}_{2}\right), 3.0\left(2 \mathrm{H}, \mathrm{m}, \mathrm{NCH}_{2}\right), 1.8-1.9(2 \mathrm{H}$, $\left.\mathrm{m}, \mathrm{CCH}_{2}\right), 1.5(9 \mathrm{H}, \mathrm{s}, \mathrm{Boc})$.

\section{5-Boc-aminopentanol (9)}

To a mixture of 5 -aminopentanol $(10.0 \mathrm{mmol})$ in acetonitrile $(50 \mathrm{~mL})$ was dropwise added di-tert-butyl dicarbonate $(10.0 \mathrm{mmol})$ dissolved in acetonitrile $(5 \mathrm{~mL})$. The mixture was stirred for $24 \mathrm{~h}$ at room temperature. Volatile components were evaporated. The residue was taken up in ethyl acetate. This was washed twice with $0.5 \mathrm{M}$ citric acid and once with $\mathrm{H}_{2} \mathrm{O}$. After the mixture was dried $\mathrm{MgSO}_{4}$, and the solvent was evaporated. The residue was vaccum desiccated overnight to yield yellow oil. 
Yield 79\%. IR ( $\mathrm{KBr}) \mathrm{cm}^{-1}:$ 1 688, 2934, 3342. ${ }^{1} \mathrm{H}$ NMR (DMSO) $\delta$ : $6.7(1 \mathrm{H}, \mathrm{s}, \mathrm{NH}), 4.4(1 \mathrm{H}, \mathrm{s}, \mathrm{OH}), 3.4\left(2 \mathrm{H}, \mathrm{t}, \mathrm{OCH}_{2}\right), 2.9(2 \mathrm{H}, \mathrm{m}$, $\left.\mathrm{NCH}_{2}\right), 1.4-1.6\left(6 \mathrm{H}, \mathrm{m}, \mathrm{CCH}_{2} \mathrm{C}\right), 1.3(9 \mathrm{H}, \mathrm{s}, \mathrm{Boc})$.

General procedure for synthesis of compounds (10-13) A solution of $\mathrm{N}$-Boc-3-bromoalkylamine 7, 8 (3.5 mmol; ) in $\mathrm{CH}_{3} \mathrm{CN}(30 \mathrm{~mL})$ was added to a mixture of $\mathbf{5 , 6}(3.0 \mathrm{mmol})$ and $\mathrm{K}_{2} \mathrm{CO}_{3}(9.0 \mathrm{mmol})$ in $\mathrm{CH}_{3} \mathrm{CN}(30 \mathrm{~mL})$. The mixture was heated under reflux for $12 \mathrm{~h}$. The inorganic material was filtered off and the solvent was evaporated in vacuo. The crude residue was extracted with $\mathrm{CH}_{3} \mathrm{Cl}$, washed with $\mathrm{H}_{2} \mathrm{O}$ and brine, dried over $\mathrm{Na}_{2} \mathrm{SO}_{4}$, and concentrated in vacuo to give oil.

\section{5-(2-Boc-aminoethoxy)-2-piperidin-1-ylmethylindan-1-} one (10)

Yield 65\%. IR (KBr) cm $\mathrm{cm}^{-1}$ : 1690, 1706, 2737, 2946, 3202. ${ }^{1} \mathrm{H}$ NMR (DMSO) $\delta: ~ 7.7 ~(1 \mathrm{H}, \mathrm{m}, \mathrm{Ar}), 6.8-6.9(2 \mathrm{H}, \mathrm{m}, \mathrm{Ar}), 6.7(1 \mathrm{H}, \mathrm{t}$, $\mathrm{NH}), 4.1\left(2 \mathrm{H}, \mathrm{t}, \mathrm{OCH}_{2}\right), 3.6\left(2 \mathrm{H}, \mathrm{d}, \mathrm{CH}_{2} \mathrm{CH}\right), 3.4\left(2 \mathrm{H}, \mathrm{d}, \mathrm{NCH}_{2}\right), 3.1-$ $3.2\left(4 \mathrm{H}, \mathrm{m}, 2 \cdot \mathrm{NCH}_{2}\right), 3.0\left(2 \mathrm{H}, \mathrm{m}, \mathrm{NHCH}_{2}\right), 2.6-2.7(1 \mathrm{H}, \mathrm{m}, \mathrm{COCH})$, 1.5-1.6 (6H, m, 3. $\left.\mathrm{CH}_{2}\right), 1.2(9 \mathrm{H}, \mathrm{s}, \mathrm{Boc})$.

\section{2-(4-benzylpiperidin-1-ylmethyl)-5-(2-Boc-}

aminoethoxy)-indan-1-one (11)

Yield 67\%. IR (KBr) cm-1: 724, 1672, 1714, 2740, 2953, 3196. ${ }^{1} \mathrm{H}$ NMR (DMSO) $\delta: ~ 7.7$ (1H, m, Ar), 7.2-7.4 (5H, m, Ar), 6.8-6.9 $(2 \mathrm{H}, \mathrm{m}, \mathrm{Ar}), 6.7(1 \mathrm{H}, \mathrm{t}, \mathrm{NH}), 4.1\left(2 \mathrm{H}, \mathrm{t}, \mathrm{OCH}_{2}\right), 3.6\left(2 \mathrm{H}, \mathrm{d}, \mathrm{CH}_{2} \mathrm{CH}\right)$, $3.4\left(2 \mathrm{H}, \mathrm{d}, \mathrm{NCH}_{2}\right), 3.1-3.2\left(4 \mathrm{H}, \mathrm{m}, 2 \cdot \mathrm{NCH}_{2}\right), 3.0\left(2 \mathrm{H}, \mathrm{m}, \mathrm{NHCH}_{2}\right)$, $2.8\left(2 \mathrm{H}, \mathrm{t}, \mathrm{ArCH}_{2}\right), 2.6-2.7(1 \mathrm{H}, \mathrm{m}, \mathrm{COCH}), 1.4-1.6\left(5 \mathrm{H}, \mathrm{m}, 2 \cdot \mathrm{CH}_{2}\right.$, $1 \cdot \mathrm{CH}), 1.2$ (9H, s, Boc).

\section{5-(3-Boc-aminopropoxy)-2-piperidin-1ylmethylindan-1-} one (12)

Yield 61\%. IR (KBr) cm ${ }^{-1}: 1689,1701,2741,2948,3215 .{ }^{1} \mathrm{H}$

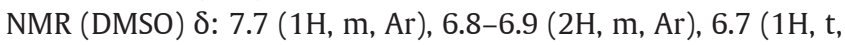
$\mathrm{NH}), 4.1\left(2 \mathrm{H}, \mathrm{t}, \mathrm{OCH}_{2}\right), 3.6\left(2 \mathrm{H}, \mathrm{d}, \mathrm{CH}_{2} \mathrm{CH}\right), 3.4\left(2 \mathrm{H}, \mathrm{d}, \mathrm{NCH}_{2}\right), 3.1-$ $3.2\left(4 \mathrm{H}, \mathrm{m}, 2 \cdot \mathrm{NCH}_{2}\right), 3.0\left(2 \mathrm{H}, \mathrm{m}, \mathrm{NHCH}_{2}\right), 2.7-2.8(1 \mathrm{H}, \mathrm{m}, \mathrm{COCH})$, 1.7-1.8 (6H, m, 3. $\left.\mathrm{CH}_{2}\right), 1.4-1.5\left(2 \mathrm{H}, \mathrm{m}, \mathrm{CCH}_{2} \mathrm{C}\right), 1.1(9 \mathrm{H}, \mathrm{s}, \mathrm{Boc})$.

\section{2-(4-benzylpiperidin-1-ylmethyl)-5-(3-Boc-}

aminopropoxy)-indan-1-one (13)

Yield 65\%. IR (KBr) cm-1: 729, 1671, 1709, 2745, 2949, 3253.

${ }^{1} \mathrm{H}$ NMR (DMSO) $\delta: ~ 7.7$ (1H, m, Ar), 7.2-7.4 (5H, m, Ar), 6.8-6.9 $(2 \mathrm{H}, \mathrm{m}, \mathrm{Ar}), 6.7(1 \mathrm{H}, \mathrm{t}, \mathrm{NH}), 4.1\left(2 \mathrm{H}, \mathrm{t}, \mathrm{OCH}_{2}\right), 3.6\left(2 \mathrm{H}, \mathrm{d}, \mathrm{CH}_{2} \mathrm{CH}\right)$, $3.4\left(2 \mathrm{H}, \mathrm{d}, \mathrm{NCH}_{2}\right), 3.1-3.2\left(4 \mathrm{H}, \mathrm{m}, 2 \cdot \mathrm{NCH}_{2}\right), 3.0\left(2 \mathrm{H}, \mathrm{m}, \mathrm{NHCH}_{2}\right)$, 2.7-2.8 (1H, m, COCH ), $2.6\left(2 \mathrm{H}, \mathrm{t}, \mathrm{ArCH}_{2}\right), 1.6-1.8\left(5 \mathrm{H}, \mathrm{m}, 2 \cdot \mathrm{CH}_{2}\right.$, $1 \cdot \mathrm{CH}), 1.3-1.4\left(2 \mathrm{H}, \mathrm{m}, \mathrm{CCH}_{2} \mathrm{C}\right), 1.2(9 \mathrm{H}, \mathrm{s}, \mathrm{Boc})$.

General procedure for synthesis of compounds (14) and (15)

TEA $(2.0 \mathrm{mmol})$ and triphenylphosphine $\left(\mathrm{Ph}_{3} \mathrm{P}\right)(2.5 \mathrm{mmol})$ were added to solution $\mathbf{5 , 6}(2.0 \mathrm{mmol})$ in anhydrous THF $(5 \mathrm{~mL})$ under argon. The mixture was stirred and cooled in a ice bath $\left(-5^{\circ} \mathrm{C}\right.$ to $\left.0^{\circ} \mathrm{C}\right)$, while diethylazodicarboxylate $(2.5 \mathrm{mmol})$ was added dropwise. After $15 \mathrm{~min}$, N-Boc-aminopentanol $(2.0 \mathrm{mmol})$ in DMF $(1 \mathrm{~mL})$ was added all at once. The reaction mixture was stirred overnight, and warmed to room temperature. The mixture was concentrated under reduced pressure. The crude product in the form of oil was purified by silica gel column chromatography, using as eluent mixtures of solvents: chloroform: methanol: $\mathrm{NH}_{3}$ saturated 9:1:0.5.
5-(5-Boc-aminopentyloxy)-2-piperidin-1-ylmethylindan1-one (14)

Yield 48\%. IR (KBr) $\mathrm{cm}^{-1}: 1686,1699,2735,2946,3210 .{ }^{1} \mathrm{H}$

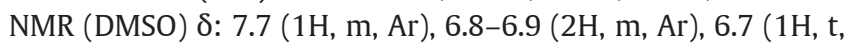
$\mathrm{NH}), 3.9\left(2 \mathrm{H}, \mathrm{t}, \mathrm{OCH}_{2}\right), 3.6\left(2 \mathrm{H}, \mathrm{d}, \mathrm{CH}_{2} \mathrm{CH}\right), 3.4\left(2 \mathrm{H}, \mathrm{d}, \mathrm{NCH}_{2}\right), 3.2-$ $3.3\left(4 \mathrm{H}, \mathrm{m}, 2 \cdot \mathrm{NCH}_{2}\right), 3.1\left(2 \mathrm{H}, \mathrm{m}, \mathrm{NHCH}_{2}\right), 2.8-2.9(1 \mathrm{H}, \mathrm{m}, \mathrm{COCH})$, 1.7-1.8 (6H, m, 3. $\left.\mathrm{CH}_{2}\right), 1.3-1.4\left(6 \mathrm{H}, \mathrm{m}, \mathrm{CCH}_{2} \mathrm{C}\right), 1.1$ (9H, s, Boc).

\section{2-(4-benzylpiperidin-1-ylmethyl)-5-(Boc-}

aminopentyloxy)-indan-1-one (15)

Yield 45\%. IR (KBr) cm ${ }^{-1}:$ 736, 1675, 1710, 2739, 2957, 3268. ${ }^{1} \mathrm{H}$ NMR (DMSO) $\delta: ~ 7.6 ~(1 \mathrm{H}, \mathrm{m}, \mathrm{Ar}), 7.2-7.4(5 \mathrm{H}, \mathrm{m}, \mathrm{Ar}), 6.8-6.9$ $(2 \mathrm{H}, \mathrm{m}, \mathrm{Ar}), 6.7(1 \mathrm{H}, \mathrm{t}, \mathrm{NH}), 3.9\left(2 \mathrm{H}, \mathrm{t}, \mathrm{OCH}_{2}\right), 3.6\left(2 \mathrm{H}, \mathrm{d}, \mathrm{CH}_{2} \mathrm{CH}\right)$, $3.4\left(2 \mathrm{H}, \mathrm{d}, \mathrm{NCH}_{2}\right), 3.2-3.3\left(4 \mathrm{H}, \mathrm{m}, 2 \cdot \mathrm{NCH}_{2}\right), 3.1\left(2 \mathrm{H}, \mathrm{m}, \mathrm{NHCH}_{2}\right)$, 2.8-2.9 (1H, m, COCH ), $2.7\left(2 \mathrm{H}, \mathrm{t}, \mathrm{ArCH}_{2}\right), 1.5-1.7\left(5 \mathrm{H}, \mathrm{m}, 2 \cdot \mathrm{CH}_{2}\right.$, $1 \cdot \mathrm{CH}), 1.3-1.4\left(6 \mathrm{H}, \mathrm{m}, \mathrm{CCH}_{2} \mathrm{C}\right), 1.1$ (9H, s, Boc).

\section{General procedure for preparation of compounds}

(16-21)

A solution of 10-15 $(0.5 \mathrm{mmol})$ in anhydrous THF $(5 \mathrm{~mL})$ was cooled to $-20^{\circ} \mathrm{C}$ and stirred for $30 \mathrm{~min}$. Etherate $\mathrm{HCl}$ was added dropwise to the reaction mixture under $\mathrm{pH}=1$. The precipitate was collected by suction filtration, washed with ether and dried in desiccator to give a white solid.

\section{5-(2-aminoethoxy)-2-piperidin-1-ylmethylindan-1-one} hydrochloride (16)

Yield $78 \%$. Mp. $145-148^{\circ} \mathrm{C}$. IR (KBr) cm ${ }^{-1}: 1697,2737,2947$, 3367. ${ }^{1} \mathrm{H}$ NMR (DMSO) $\delta: ~ 7.7 ~(1 \mathrm{H}, \mathrm{s}, \mathrm{Ar}$ ), 6.8-6.9 (2H, m, Ar), 4.1 $\left(2 \mathrm{H}, \mathrm{t}, \mathrm{OCH}_{2}\right), 3.6\left(2 \mathrm{H}, \mathrm{d}, \mathrm{CH}_{2} \mathrm{CH}\right), 3.4\left(2 \mathrm{H}, \mathrm{d}, \mathrm{NCH}_{2}\right), 3.1-3.2(4 \mathrm{H}$, $\left.\mathrm{m}, 2 \cdot \mathrm{NCH}_{2}\right), 3.0\left(2 \mathrm{H}, \mathrm{m}, \mathrm{NHCH}_{2}\right), 2.6-2.7(1 \mathrm{H}, \mathrm{m}, \mathrm{COCH}), 2.3(2 \mathrm{H}$, s, $\left.\mathrm{NH}_{2}\right), 1.5-1.6\left(6 \mathrm{H}, \mathrm{m}, 3 \cdot \mathrm{CH}_{2}\right)$. Anal. calc. for $\mathrm{C}_{17} \mathrm{H}_{25} \mathrm{ClN}_{2} \mathrm{O}_{2}(\%)$ : C 62.86, H 7.76, N 8.62; Found (\%): C 62.38, H 7.52, N 8.41.

\section{5-(2-aminoethoxy)- 2-(4-benzylpiperidin-1-ylmethyl)-} indan-1-one hydrochloride (17)

Yield $70 \%$ Mp. $168-171^{\circ} \mathrm{C}$. IR $(\mathrm{KBr}) \mathrm{cm}^{-1}: 738,1695,2694$, 2944, 3370. ${ }^{1} \mathrm{H}$ NMR (DMSO) $\delta: ~ 7.7 ~(1 \mathrm{H}, \mathrm{s}, \mathrm{Ar}), 7.2-7.4(5 \mathrm{H}, \mathrm{m}$, Ar), 6.8-6.9 (2H, m, Ar), $4.1\left(2 \mathrm{H}, \mathrm{t}, \mathrm{OCH}_{2}\right), 3.6\left(2 \mathrm{H}, \mathrm{d}, \mathrm{CH}_{2} \mathrm{CH}\right), 3.4$ $\left(2 \mathrm{H}, \mathrm{d}, \mathrm{NCH}_{2}\right), 3.1-3.2\left(4 \mathrm{H}, \mathrm{m}, 2 \cdot \mathrm{NCH}_{2}\right), 3.0\left(2 \mathrm{H}, \mathrm{m}, \mathrm{NHCH}_{2}\right), 2.8$ $\left(2 \mathrm{H}, \mathrm{t}, \mathrm{ArCH}_{2}\right), 2.5-2.6(1 \mathrm{H}, \mathrm{m}, \mathrm{COCH}), 2.1\left(2 \mathrm{H}, \mathrm{s}, \mathrm{NH}_{2}\right), 1.2-1.4$ $\left(5 \mathrm{H}, \mathrm{m}, 2 \cdot \mathrm{CH}_{2}, 1 \cdot \mathrm{CH}\right)$. Anal. calc. for $\mathrm{C}_{24} \mathrm{H}_{31} \mathrm{ClN}_{2} \mathrm{O}_{2}(\%): \mathrm{C} 69.46, \mathrm{H}$ 7.53, N 6.75; Found (\%): C 69.11, H 7.23, N 6.61.

\section{5-(3-aminopropoxy)-2-piperidin-1-ylmethylindan-1-one} hydrochloride (18)

Yield 76\%. Mp. $139-142^{\circ} \mathrm{C}$. IR (KBr) $\mathrm{cm}^{-1}: 1689,2740,2948$, 3360. ${ }^{1} \mathrm{H}$ NMR (DMSO) $\delta: 7.7$ (1H, s, Ar), 6.8-6.9 (2H, m, Ar), 4.1 $\left(2 \mathrm{H}, \mathrm{t}, \mathrm{OCH}_{2}\right), 3.6\left(2 \mathrm{H}, \mathrm{d}, \mathrm{CH}_{2} \mathrm{CH}\right), 3.4\left(2 \mathrm{H}, \mathrm{d}, \mathrm{NCH}_{2}\right), 3.1-3.2(4 \mathrm{H}$, $\left.\mathrm{m}, 2 \cdot \mathrm{NCH}_{2}\right), 3.0\left(2 \mathrm{H}, \mathrm{m}, \mathrm{NHCH}_{2}\right), 2.7-2.8(1 \mathrm{H}, \mathrm{m}, \mathrm{COCH}), 2.3(2 \mathrm{H}$, s, $\left.\mathrm{NH}_{2}\right), 1.6-1.7\left(6 \mathrm{H}, \mathrm{m}, 3 \cdot \mathrm{CH}_{2}\right), 1.2-1.4\left(2 \mathrm{H}, \mathrm{m}, \mathrm{CCH}_{2} \mathrm{C}\right)$. Anal. calc. for $\mathrm{C}_{18} \mathrm{H}_{27} \mathrm{ClN}_{2} \mathrm{O}_{2}$ (\%): C 63.80, $\mathrm{H} \mathrm{8.03,N} \mathrm{8.27;} \mathrm{Found} \mathrm{( \% ):} \mathrm{C}$ 63.27, H 7.85, N 8.11.

\section{5-(3-aminopropoxy)- 2-(4-benzylpiperidin-1-ylmethyl)-} indan-1-one hydrochloride (19)

Yield $76 \%$ Mp. $165-167^{\circ} \mathrm{C}$. IR $(\mathrm{KBr}) \mathrm{cm}^{-1}: 730,1687,2732$, 2949, 3363. ${ }^{1} \mathrm{H}$ NMR (DMSO) $\delta: ~ 7.7 ~(1 \mathrm{H}, \mathrm{s}, \mathrm{Ar}), 7.2-7.4(5 \mathrm{H}, \mathrm{m}$, Ar), 6.8-6.9 (2H, m, Ar), $4.1\left(2 \mathrm{H}, \mathrm{t}, \mathrm{OCH}_{2}\right), 3.6\left(2 \mathrm{H}, \mathrm{d}, \mathrm{CH}_{2} \mathrm{CH}\right), 3.4$ $\left(2 \mathrm{H}, \mathrm{d}, \mathrm{NCH}_{2}\right), 3.1-3.2\left(4 \mathrm{H}, \mathrm{m}, 2 \cdot \mathrm{NCH}_{2}\right), 3.0\left(2 \mathrm{H}, \mathrm{m}, \mathrm{NHCH}_{2}\right), 2.7-$ $2.8(1 \mathrm{H}, \mathrm{m}, \mathrm{COCH}), 2.6\left(2 \mathrm{H}, \mathrm{t}, \mathrm{ArCH}_{2}\right), 2.2\left(2 \mathrm{H}, \mathrm{s}, \mathrm{NH}_{2}\right), 1.5-1.8$ $\left(5 \mathrm{H}, \mathrm{m}, 2 \cdot \mathrm{CH}_{2}, 1 \cdot \mathrm{CH}\right), 1.2-1.3\left(2 \mathrm{H}, \mathrm{m}, \mathrm{CCH}_{2} \mathrm{C}\right)$. Anal. calc. for 
$\mathrm{C}_{25} \mathrm{H}_{33} \mathrm{ClN}_{2} \mathrm{O}_{2}$ (\%): C 69.99, H 7.75, N 6.53; Found (\%): C 69.58, H 7.43, N 6.32 .

5-(5-aminopentyloxy)-2-piperidin-1-ylmethylindan-1one hydrochloride (20)

Yield 69\%. Mp. $125-128^{\circ} \mathrm{C}$. IR (KBr) $\mathrm{cm}^{-1}: 1696,2735,2946$, 3365. ${ }^{1} \mathrm{H}$ NMR (DMSO) 8: 7.7 (1H, s, Ar), 6.8-6.9 (2H, m, Ar), 3.9 $\left(2 \mathrm{H}, \mathrm{t}, \mathrm{OCH}_{2}\right), 3.6\left(2 \mathrm{H}, \mathrm{d}, \mathrm{CH}_{2} \mathrm{CH}\right), 3.4\left(2 \mathrm{H}, \mathrm{d}, \mathrm{NCH}_{2}\right), 3.2-3.3(4 \mathrm{H}$, $\left.\mathrm{m}, 2 \cdot \mathrm{NCH}_{2}\right), 3.1\left(2 \mathrm{H}, \mathrm{m}, \mathrm{NHCH}_{2}\right), 2.8-2.9(1 \mathrm{H}, \mathrm{m}, \mathrm{COCH}), 2.2(2 \mathrm{H}$, s, $\left.\mathrm{NH}_{2}\right), 1.6-1.7\left(6 \mathrm{H}, \mathrm{m}, 3 \cdot \mathrm{CH}_{2}\right), 1.2-1.4\left(6 \mathrm{H}, \mathrm{m}, \mathrm{CCH}_{2} \mathrm{C}\right)$. Anal. calc. for $\mathrm{C}_{20} \mathrm{H}_{31} \mathrm{ClN}_{2} \mathrm{O}_{2}$ (\%): C 65.47, H 8.52, N 7.63; Found (\%): C 65.09, H 8.34, N 7.41.

5-(5-aminopentyloxy)-2-(4-benzylpiperidin-1-ylmethyl)indan-1-one hydrochloride (21)

Yield $74 \%$. Mp. $160-163^{\circ} \mathrm{C}$. IR (KBr) $\mathrm{cm}^{-1}$ : 736, 1700,2735 , 2948, 3375. ${ }^{1} \mathrm{H}$ NMR (DMSO) 8: $7.6(1 \mathrm{H}, \mathrm{s}, \mathrm{Ar}), 7.2-7.4(5 \mathrm{H}, \mathrm{m}$, $\mathrm{Ar}), 6.8-6.9(2 \mathrm{H}, \mathrm{m}, \mathrm{Ar}), 3.9\left(2 \mathrm{H}, \mathrm{t}, \mathrm{OCH}_{2}\right), 3.6\left(2 \mathrm{H}, \mathrm{d}, \mathrm{CH}_{2} \mathrm{CH}\right), 3.4$ $\left(2 \mathrm{H}, \mathrm{d}, \mathrm{NCH}_{2}\right), 3.2-3.3\left(4 \mathrm{H}, \mathrm{m}, 2 \cdot \mathrm{NCH}_{2}\right), 3.1\left(2 \mathrm{H}, \mathrm{m}, \mathrm{NHCH}_{2}\right), 2.8-$ $2.9(1 \mathrm{H}, \mathrm{m}, \mathrm{COCH}), 2.7\left(2 \mathrm{H}, \mathrm{t}, \mathrm{ArCH}_{2}\right), 2.2\left(2 \mathrm{H}, \mathrm{s}, \mathrm{NH}_{2}\right), 1.3-1.7$ $\left(5 \mathrm{H}, \mathrm{m}, 2 \cdot \mathrm{CH}_{2}, 1 \cdot \mathrm{CH}\right), 1.2-1.3\left(2 \mathrm{H}, \mathrm{m}, \mathrm{CCH}_{2} \mathrm{C}\right)$. Anal. calc. for $\mathrm{C}_{27} \mathrm{H}_{37} \mathrm{ClN}_{2} \mathrm{O}_{2}$ (\%): C 70.95, H 8.16, N 6.13; Found (\%): C 70.61, H 7.96, N 5.87 .

\section{General procedure for preparation of compounds}

(22-27)

$1,1^{\prime}$-carbonyldiimidazole $(1.0 \mathrm{mmol})$ was added to solution $\mathbf{2}$ in anhydrous THF $(10 \mathrm{~mL})$.

The mixture was stirred for $4 \mathrm{~h}$ in room temperature. Next, the corresponding amine 16-21 $(1.0 \mathrm{mmol})$ and TEA (1.0 mmol) were added. The resulting amber solution was stirred for $20 \mathrm{~h}$. The solvent was evaporated under reduced pressure, water was added, and the resultant mixture was extracted twice with $\mathrm{CH}_{3} \mathrm{Cl}$. The combined organic extracts were washed with brine and then dried with anhydrous $\mathrm{Na}_{2} \mathrm{SO}_{4}$. The solvent was evaporated under reduce pressure and the residue was purified by crystallization from methanol.

6-Boc-hydrazino-N-[2-(1-oxo-2-piperidin-1-

ylmethylindan-5-yloxy)-ethyl]-nicotinamide (22)

Yield $45 \%$. Mp. $174-177^{\circ} \mathrm{C}$. IR (KBr) cm ${ }^{-1}: 1641,1696,1711$, 2974, 3375. ${ }^{1} \mathrm{H}$ NMR (DMSO) $\delta: 8.7$ (1H, s, NHC), 8.5 (1H, s, Ar), $8.2(1 \mathrm{H}, \mathrm{t}, \mathrm{COCN}), 7.9(1 \mathrm{H}, \mathrm{d}, \mathrm{Ar}), 7.6(1 \mathrm{H}, \mathrm{s}, \mathrm{Ar}), 6.8-6.9(2 \mathrm{H}, \mathrm{m}$, $\mathrm{Ar}), 6.5(1 \mathrm{H}, \mathrm{d}, \mathrm{Ar}), 4.1\left(2 \mathrm{H}, \mathrm{t}, \mathrm{OCH}_{2}\right), 3.6\left(2 \mathrm{H}, \mathrm{d}, \mathrm{CH}_{2} \mathrm{CH}\right), 3.4(2 \mathrm{H}$, d, $\left.\mathrm{NCH}_{2}\right), 3.3(1 \mathrm{H}, \mathrm{d}, \mathrm{CNH}), 3.1-3.2\left(4 \mathrm{H}, \mathrm{m}, 2 \cdot \mathrm{NCH}_{2}\right), 3.0(2 \mathrm{H}, \mathrm{m}$, $\left.\mathrm{NHCH}_{2}\right), 2.6-2.7(1 \mathrm{H}, \mathrm{m}, \mathrm{COCH}), 1.5-1.6\left(6 \mathrm{H}, \mathrm{m}, 3 \cdot \mathrm{CH}_{2}\right), 1.2(9 \mathrm{H}$, s, Boc). Anal. calc. for $\mathrm{C}_{28} \mathrm{H}_{37} \mathrm{~N}_{5} \mathrm{O}_{5}$ (\%): C 64.23, H 7.12, N 13.37; Found (\%): C 63.87, H 7.23, N 13.15 .

\section{N-\{-[2-(4-benzylpiperidin-1-ylmethyl)-1-oxoindan-5-}

yloxy]-ethyl\}-6-Boc-hydrazinonicotinamide (23)

Yield $47 \%$. Mp. $192-195^{\circ} \mathrm{C}$. IR (KBr) cm ${ }^{-1}: 742,1601,1695$, 1702, 2989, 3278. ${ }^{1} \mathrm{H}$ NMR (DMSO) $\delta: 8.8$ (1H, s, NHC), $8.4(1 \mathrm{H}$, s, Ar), $8.3(1 \mathrm{H}, \mathrm{s}, \mathrm{CONH}), 7.8(1 \mathrm{H}, \mathrm{d}, \mathrm{Ar}), 7.7(1 \mathrm{H}, \mathrm{s}, \mathrm{Ar}), 7.2-7.4$ (5H, m, Ar), 6.8-6.9 (2H, m, Ar), $6.5(1 \mathrm{H}, \mathrm{d}, \mathrm{Ar}), 4.1\left(2 \mathrm{H}, \mathrm{t}, \mathrm{OCH}_{2}\right)$, $3.6\left(2 \mathrm{H}, \mathrm{d}, \mathrm{CH}_{2} \mathrm{CH}\right), 3.4\left(2 \mathrm{H}, \mathrm{d}, \mathrm{NCH}_{2}\right), 3.3(1 \mathrm{H}, \mathrm{s}, \mathrm{CNH}), 3.1-3.2$ $\left(4 \mathrm{H}, \mathrm{m}, 2 \cdot \mathrm{NCH}_{2}\right), 3.0\left(2 \mathrm{H}, \mathrm{m}, \mathrm{NHCH}_{2}\right), 2.8\left(2 \mathrm{H}, \mathrm{t}, \mathrm{ArCH}_{2}\right), 2.5-2.6$ $(1 \mathrm{H}, \mathrm{m}, \mathrm{COCH}), 1.3-1.6\left(5 \mathrm{H}, \mathrm{m}, 2 \cdot \mathrm{CH}_{2}, 1 \cdot \mathrm{CH}\right) .1,2(9 \mathrm{H}, \mathrm{s}, \mathrm{Boc})$. Anal. calc. for $\mathrm{C}_{35} \mathrm{H}_{43} \mathrm{~N}_{5} \mathrm{O}_{5}$ (\%): C 68.49, $\mathrm{H}$ 7.06, N 11.41; Found (\%): C 67.96, H 6.78, N 11.34.
6-Boc-hydrazino-N-[3-(1-oxo-2-piperidin-1ylmethylindan-5-yloxy)-propyl]-nicotinamide (24) Yield $50 \%$. Mp. $176-179^{\circ} \mathrm{C}$. IR (KBr) $\mathrm{cm}^{-1}: 1640,1694,1710$,

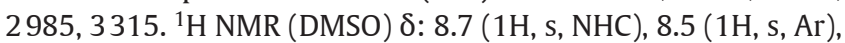
$8.1(1 \mathrm{H}, \mathrm{t}, \mathrm{CONH}), 7.8(1 \mathrm{H}, \mathrm{d}, \mathrm{Ar}), 7.6(1 \mathrm{H}, \mathrm{s}, \mathrm{Ar}), 6.8-6.9(2 \mathrm{H}, \mathrm{m}$, $\mathrm{Ar}), 6.6(1 \mathrm{H}, \mathrm{d}, \mathrm{Ar}), 4.1\left(2 \mathrm{H}, \mathrm{t}, \mathrm{OCH}_{2}\right), 3.7\left(2 \mathrm{H}, \mathrm{d}, \mathrm{CH}_{2} \mathrm{CH}\right), 3.4(2 \mathrm{H}$, d, $\left.\mathrm{NCH}_{2}\right), 3.3(1 \mathrm{H}, \mathrm{d}, \mathrm{CNH}), 3.1-3.2\left(4 \mathrm{H}, \mathrm{m}, 2 \cdot \mathrm{NCH}_{2}\right), 3.0(2 \mathrm{H}, \mathrm{m}$, $\left.\mathrm{NHCH}_{2}\right), 2.6-2.7(1 \mathrm{H}, \mathrm{m}, \mathrm{COCH}), 1.6-1.7\left(6 \mathrm{H}, \mathrm{m}, 3 \cdot \mathrm{CH}_{2}\right), 1.2-1.4$ $\left(2 \mathrm{H}, \mathrm{m}, \mathrm{CCH}_{2} \mathrm{C}\right), 1.0(9 \mathrm{H}, \mathrm{s}, \mathrm{Boc})$. Anal. calc. for $\mathrm{C}_{29} \mathrm{H}_{39} \mathrm{~N}_{5} \mathrm{O}_{5}(\%)$ : C 64.78, H 7.31, N 13.03; Found (\%): C 64.97, H 7.28, N 13.23.

\section{N-\{3-[2-(4-benzylpiperidin-1-ylmethyl)-1-oxoindan-5-}

yloxy]-propyl\}-6-Boc-hydrazinonicotinamide (25)

Yield $49 \%$. Mp. $194-197^{\circ} \mathrm{C}$. IR (KBr) cm ${ }^{-1}: 734,1595,1692$, 1707, 2990, 3287. ${ }^{1} \mathrm{H}$ NMR (DMSO) $8: 8.8$ (1H, s, NHC), $8.4(1 \mathrm{H}$, s, Ar), $8.1(1 \mathrm{H}, \mathrm{s}, \mathrm{CONH}), 7.9(1 \mathrm{H}, \mathrm{d}, \mathrm{Ar}), 7.6(1 \mathrm{H}, \mathrm{s}, \mathrm{Ar}), 7.2-7.4$ $(5 \mathrm{H}, \mathrm{m}, \mathrm{Ar}), 6.8-6.9(2 \mathrm{H}, \mathrm{m}, \mathrm{Ar}), 6.5(1 \mathrm{H}, \mathrm{d}, \mathrm{Ar}), 4.1\left(2 \mathrm{H}, \mathrm{t}, \mathrm{OCH}_{2}\right)$, $3.6\left(2 \mathrm{H}, \mathrm{d}, \mathrm{CH}_{2} \mathrm{CH}\right), 3.4\left(2 \mathrm{H}, \mathrm{d}, \mathrm{NCH}_{2}\right), 3.3(1 \mathrm{H}, \mathrm{d}, \mathrm{CNH}), 3.1-3.2$ $\left(4 \mathrm{H}, \mathrm{m}, 2 \cdot \mathrm{NCH}_{2}\right), 3.0\left(2 \mathrm{H}, \mathrm{k}, \mathrm{NHCH}_{2}\right), 2.7-2.8(1 \mathrm{H}, \mathrm{m}, \mathrm{COCH}), 2.6$ $\left(2 \mathrm{H}, \mathrm{t}, \mathrm{ArCH}_{2}\right), 1.7-1.9\left(5 \mathrm{H}, \mathrm{m}, 2 \cdot \mathrm{CH}_{2}, 1 \cdot \mathrm{CH}\right), 1.2-1.3(2 \mathrm{H}, \mathrm{m}$, $\left.\mathrm{CCH}_{2} \mathrm{C}\right), 1.0(9 \mathrm{H}, \mathrm{s}, \mathrm{Boc})$. Anal. calc. for $\mathrm{C}_{36} \mathrm{H}_{45} \mathrm{~N}_{5} \mathrm{O}_{5}$ (\%): $\mathrm{C} 68.88, \mathrm{H}$ 7.23, N 11.16; Found (\%): C 68.41, H 6.91, N 10.77.

\section{6-Boc-hydrazino-N-[5-(1-oxo-2-piperidin-1-}

ylmethylindan-5-yloxy)-pentyl]-nicotinamide (26)

Yield $45 \%$. Mp. $181-183^{\circ} \mathrm{C}$. IR (KBr) $\mathrm{cm}^{-1}: 1639,1697,1706$, 2991, 3352. ${ }^{1} \mathrm{H}$ NMR (DMSO) $\delta: 8.7$ (1H, s, NHC), 8.5 (1H, s, Ar), $8.2(1 \mathrm{H}, \mathrm{t}, \mathrm{CONH}), 7.9(1 \mathrm{H}, \mathrm{d}, \mathrm{Ar}), 7.6(1 \mathrm{H}, \mathrm{s}, \mathrm{Ar}), 6.8-6.9(2 \mathrm{H}, \mathrm{m}$, $\mathrm{Ar}), 6.5(1 \mathrm{H}, \mathrm{d}, \mathrm{Ar}), 3.9\left(2 \mathrm{H}, \mathrm{t}, \mathrm{OCH}_{2}\right), 3.6\left(2 \mathrm{H}, \mathrm{d}, \mathrm{CH}_{2} \mathrm{CH}\right), 3.4(2 \mathrm{H}$, d, $\left.\mathrm{NCH}_{2}\right), 3.3(1 \mathrm{H}, \mathrm{d}, \mathrm{CNH}), 3.1-3.2\left(4 \mathrm{H}, \mathrm{m}, 2 \cdot \mathrm{NCH}_{2}\right), 3.0(2 \mathrm{H}, \mathrm{m}$, $\left.\mathrm{NHCH}_{2}\right), 2.8-2.9(1 \mathrm{H}, \mathrm{m}, \mathrm{COCH}), 1.6-1.7\left(6 \mathrm{H}, \mathrm{m}, 3 \cdot \mathrm{CH}_{2}\right), 1.1-1.3$ $\left(6 \mathrm{H}, \mathrm{m}, \mathrm{CCH}_{2} \mathrm{C}\right), 1.0(9 \mathrm{H}, \mathrm{s}, \mathrm{Boc})$. Anal. calc. for $\mathrm{C}_{31} \mathrm{H}_{43} \mathrm{~N}_{5} \mathrm{O}_{5}$ (\%): C 65.82, H 7.66, N 12.38; Found (\%): C 65.46, H 7.50, N 12.21.

$\mathrm{N}$-\{5-[2-(4-benzylpiperidin-1-ylmethyl)-1-oxoindan-5yloxy]-pentyl\}-6-Boc-hydrazinonicotinamide (27) Yield $42 \%$. Mp. 201-204 ${ }^{\circ} \mathrm{C}$. IR (KBr) $\mathrm{cm}^{-1}: 732,1601,1690$, $1711,2985,3297 .{ }^{1} \mathrm{H}$ NMR (DMSO) $\delta: ~ 8.8$ (1H, s, NHC), $8.4(1 \mathrm{H}$, s, $\operatorname{Ar}), 8.1(1 \mathrm{H}, \mathrm{t}, \mathrm{CONH}), 7.9(1 \mathrm{H}, \mathrm{d}, \mathrm{Ar}), 7.6(1 \mathrm{H}, \mathrm{s}, \mathrm{Ar}), 7.2-7.4$ (5H, m, Ar), 6.8-6.9 (2H, m, Ar), 6.4 (1H, d, Ar), $3.9\left(2 \mathrm{H}, \mathrm{t}, \mathrm{OCH}_{2}\right)$, $3.7\left(2 \mathrm{H}, \mathrm{d}, \mathrm{CH}_{2} \mathrm{CH}\right), 3.5\left(2 \mathrm{H}, \mathrm{d}, \mathrm{NCH}_{2}\right), 3.2-3.4\left(5 \mathrm{H}, \mathrm{m}, 2 \cdot \mathrm{NCH}_{2}\right.$, $\mathrm{CNH}), 3.1\left(2 \mathrm{H}, \mathrm{m}, \mathrm{NHCH}_{2}\right), 2.8-2.9(1 \mathrm{H}, \mathrm{m}, \mathrm{COCH}), 2.7(2 \mathrm{H}, \mathrm{t}$, $\left.\mathrm{ArCH}_{2}\right), 1.6-1.8\left(5 \mathrm{H}, \mathrm{m}, 2 \cdot \mathrm{CH}_{2}, 1 \cdot \mathrm{CH}\right), 1.2-1.3\left(6 \mathrm{H}, \mathrm{m}, \mathrm{CCH}_{2} \mathrm{C}\right)$, 1.1 (9H, s, Boc). Anal. calc. for $\mathrm{C}_{38} \mathrm{H}_{49} \mathrm{~N}_{5} \mathrm{O}_{5}$ (\%): C 69.59, $\mathrm{H} 7.53, \mathrm{~N}$ 10.68; Found (\%): C 69.14, H 7.38, N 10.31.

\section{General procedure for preparation of compounds (28-33)}

$0.5 \mathrm{mmol}$ 22-27 was dissolved in anhydous THF $(5 \mathrm{~mL})$ and ether saturated $\mathrm{HCl}$ was dropwise added. Mixture was stirred in room temperature and a percipitate was formed. The percipitate was isolated and the solid was washed with ether.

6-hydrazino-N-[2-(1-oxo-2-piperidin-1-ylmethylindan-5yloxy)-ethyl]-nicotinamide hydrochloride (28)

Yield 65\%. Mp. $175-178^{\circ} \mathrm{C}$. IR (KBr) $\mathrm{cm}^{-1}: 1639,1696,2787$, 2974, 3154. ${ }^{1} \mathrm{H}$ NMR (DMSO): 8.5 (1H, s, Ar), 8.2 (1H, t, CNHC), $7.9(1 \mathrm{H}, \mathrm{d}, \mathrm{Ar}), 7.6(1 \mathrm{H}, \mathrm{s}, \mathrm{Ar}), 6.8-6.9(2 \mathrm{H}, \mathrm{m}, \mathrm{Ar}), 6.5(1 \mathrm{H}, \mathrm{d}, \mathrm{Ar})$, $4.1\left(2 \mathrm{H}, \mathrm{t}, \mathrm{OCH}_{2}\right), 3.6\left(2 \mathrm{H}, \mathrm{d}, \mathrm{CH}_{2} \mathrm{CH}\right), 3.4\left(2 \mathrm{H}, \mathrm{d}, \mathrm{NCH}_{2}\right), 3.3(1 \mathrm{H}$, d, $\mathrm{CNH}), 3.1-3.2\left(4 \mathrm{H}, \mathrm{m}, 2 \cdot \mathrm{NCH}_{2}\right), 3.0\left(2 \mathrm{H}, \mathrm{m}, \mathrm{NHCH}_{2}\right), 2.6-2.7$ $(1 \mathrm{H}, \mathrm{m}, \mathrm{COCH}), 2.2\left(2 \mathrm{H}, \mathrm{d}, \mathrm{NH}_{2}\right), 1.5-1.6\left(6 \mathrm{H}, \mathrm{m}, 2 \cdot \mathrm{CH}_{2}, 1 \cdot \mathrm{CH}\right)$. 
MS (FAB) (m/z): $425(\mathrm{M}+1)$. Anal. calc. for $\mathrm{C}_{23} \mathrm{H}_{30} \mathrm{ClN}_{5} \mathrm{O}_{3}$ (\%): C 60.06, H 6.57, N 15.23; Found (\%): C 59.71, H 6.40, N 14.92.

$\mathrm{N}$-\{2-[2-(4-benzylpiperidin-1-ylmethyl)-1-oxoindan-5yloxy]-ethyl\}-6-hydrazinonicotinamide hydrochloride (29)

Yield $62 \%$. Mp. $185-188^{\circ} \mathrm{C}$. IR (KBr) $\mathrm{cm}^{-1}: 740,1633,1696$, 2693, 2989, 3252. ${ }^{1} \mathrm{H}$ NMR (DMSO) $\delta: ~ 8.4(1 \mathrm{H}, \mathrm{s}, \mathrm{Ar}), 8.3(1 \mathrm{H}, \mathrm{s}$, CONH), 7.8 (1H, d, Ar), 7.7 (1H, s, Ar), 7.2-7.4 (5H, m, Ar), 6.8-6.9 $(2 \mathrm{H}, \mathrm{m}, \mathrm{Ar}), 6.5(1 \mathrm{H}, \mathrm{d}, \mathrm{Ar}), 4.1\left(2 \mathrm{H}, \mathrm{t}, \mathrm{OCH}_{2}\right), 3.6\left(2 \mathrm{H}, \mathrm{d}, \mathrm{CH}_{2} \mathrm{CH}\right)$, $3.4\left(2 \mathrm{H}, \mathrm{d}, \mathrm{NCH}_{2}\right), 3.3(1 \mathrm{H}, \mathrm{s}, \mathrm{CNH}), 3.1-3.2\left(4 \mathrm{H}, \mathrm{m}, 2 \cdot \mathrm{NCH}_{2}\right), 3.0$ $\left(2 \mathrm{H}, \mathrm{m}, \mathrm{NHCH}_{2}\right), 2.8\left(2 \mathrm{H}, \mathrm{t}, \mathrm{ArCH}_{2}\right), 2.5-2.6(1 \mathrm{H}, \mathrm{m}, \mathrm{COCH}), 2.2$ (2H, d, $\left.\mathrm{NH}_{2}\right), 1.2-1.4\left(5 \mathrm{H}, \mathrm{m}, 2 \cdot \mathrm{CH}_{2}, 1 \cdot \mathrm{CH}\right)$. MS (FAB) (m/z): 516 $(\mathrm{M}+1)$. Anal. calc. for $\mathrm{C}_{30} \mathrm{H}_{36} \mathrm{ClN}_{5} \mathrm{O}_{3}$ (\%): C 65.50, H 6.60, N 12.73; Found (\%): C 65.28, H 6.46, N 12.59 .

6-hydrazino-N-[3-(1-oxo-2-piperidin-1-ylmethylindan-5yloxy)-propyl]-nicotinamide hydrochloride (30)

Yield $63 \%$. Mp. $170-173^{\circ} \mathrm{C}$. IR ( $\left.\mathrm{KBr}\right) \mathrm{cm}^{-1}: 1642,1690,2698$, 2985, 3159. ${ }^{1} \mathrm{H}$ NMR (DMSO) $\delta: 8.5$ (1H, s, Ar), 8.2 (1H, t, CNHC), $7.9(1 \mathrm{H}, \mathrm{d}, \mathrm{Ar}), 7.6(1 \mathrm{H}, \mathrm{s}, \mathrm{Ar}), 6.8-6.9(2 \mathrm{H}, \mathrm{m}, \mathrm{Ar}), 6.5(1 \mathrm{H}, \mathrm{d}, \mathrm{Ar})$, $4.1\left(2 \mathrm{H}, \mathrm{t}, \mathrm{OCH}_{2}\right), 3.6\left(2 \mathrm{H}, \mathrm{d}, \mathrm{CH}_{2} \mathrm{CH}\right), 3.4\left(2 \mathrm{H}, \mathrm{d}, \mathrm{NCH}_{2}\right), 3.3(1 \mathrm{H}$, d, $\mathrm{CNH}) 3.1-3.2\left(4 \mathrm{H}, \mathrm{m}, 2 \cdot \mathrm{NCH}_{2}\right), 3.0\left(2 \mathrm{H}, \mathrm{m}, \mathrm{NHCH}_{2}\right), 2.6-2.7$ $(1 \mathrm{H}, \mathrm{m}, \mathrm{COCH}), 2.3\left(2 \mathrm{H}, \mathrm{d}, \mathrm{NH}_{2}\right), 1.6-1.7\left(6 \mathrm{H}, \mathrm{m}, 3 \cdot \mathrm{CH}_{2}\right), 1.2-1.4$ $\left(2 \mathrm{H}, \mathrm{m}, \mathrm{CCH}_{2} \mathrm{C}\right)$. MS $(\mathrm{FAB})(\mathrm{m} / \mathrm{z}): 439(\mathrm{M}+1)$. Anal. calc. for $\mathrm{C}_{24} \mathrm{H}_{32} \mathrm{ClN}_{5} \mathrm{O}_{3}$ (\%): C 60.81, H 6.80, N 14.78; Found (\%): C 60.49, H 6.64, N 14.53.

N-\{3-[2-(4-benzylpiperidin-1-ylmethyl)-1-oxoindan-5yloxy]-propyl]-6-hydrazinonicotinamide hydrochloride (31)

Yield 59\%. Mp. $180-183^{\circ} \mathrm{C}$. IR (KBr) $\mathrm{cm}^{-1}: 739,1642,1692$, 2698, 2991, 3242. ${ }^{1} \mathrm{H}$ NMR (DMSO) $\delta: ~ 8.4 ~(1 \mathrm{H}, \mathrm{s}, \mathrm{Ar}), 8.3(1 \mathrm{H}, \mathrm{s}$, CONH), 7.8 (1H, d, Ar), 7.7 (1H, s, Ar), 7.2-7.4 (5H, m, Ar), 6.8-6.9 $(2 \mathrm{H}, \mathrm{m}, \mathrm{Ar}), 6.5(1 \mathrm{H}, \mathrm{d}, \mathrm{Ar}), 4.1\left(2 \mathrm{H}, \mathrm{t}, \mathrm{OCH}_{2}\right), 3.6\left(2 \mathrm{H}, \mathrm{d}, \mathrm{CH}_{2} \mathrm{CH}\right)$, $3.4\left(2 \mathrm{H}, \mathrm{d}, \mathrm{NCH}_{2}\right), 3.3(1 \mathrm{H}, \mathrm{s}, \mathrm{CNH}), 3.1-3.2\left(4 \mathrm{H}, \mathrm{m}, 2 \cdot \mathrm{NCH}_{2}\right), 3.0$ $\left(2 \mathrm{H}, \mathrm{m}, \mathrm{NHCH}_{2}\right), 2.8\left(2 \mathrm{H}, \mathrm{t}, \mathrm{ArCH}_{2}\right), 2.5-2.6(1 \mathrm{H}, \mathrm{m}, \mathrm{COCH}), 2.1$ $\left(2 \mathrm{H}, \mathrm{d}, \mathrm{NH}_{2}\right), 1.3-1.7\left(5 \mathrm{H}, \mathrm{m}, 2 \cdot \mathrm{CH}_{2}, 1 \cdot \mathrm{CH}\right), 1.1-1.2(2 \mathrm{H}, \mathrm{m}$, $\left.\mathrm{CCH}_{2} \mathrm{C}\right)$. MS (FAB) (m/z): $529(\mathrm{M}+1)$. Anal. calc. for $\mathrm{C}_{31} \mathrm{H}_{38} \mathrm{ClN}_{5} \mathrm{O}_{3}$ (\%): C 66.00, H 6.79, N 12.41; Found (\%): C 65.56, H 6.61, N 12.27.

6-hydrazino-N-[5-(1-oxo-2-piperidin-1-ylmethylindan-5yloxy)-pentyl]-nicotinamide hydrochloride (32)

Yield 59\%. Mp. $161-164^{\circ} \mathrm{C}$. IR ( $\left.\mathrm{KBr}\right) \mathrm{cm}^{-1}: 1644,1697,2734$, 2990, 3 187. ${ }^{1} \mathrm{H}$ NMR (DMSO) $8: 8.5$ (1H, s, Ar), 8.2 (1H, t, CNHC), $7.9(1 \mathrm{H}, \mathrm{d}, \mathrm{Ar}), 7.6(1 \mathrm{H}, \mathrm{s}, \mathrm{Ar}), 6.8-6.9(2 \mathrm{H}, \mathrm{m}, \mathrm{Ar}), 6.5(1 \mathrm{H}, \mathrm{d}, \mathrm{Ar})$, $4.0\left(2 \mathrm{H}, \mathrm{t}, \mathrm{OCH}_{2}\right), 3.6\left(2 \mathrm{H}, \mathrm{d}, \mathrm{CH}_{2} \mathrm{CH}\right), 3.4\left(2 \mathrm{H}, \mathrm{d}, \mathrm{NCH}_{2}\right), 3.3(1 \mathrm{H}$, d, $\mathrm{CNH}), 3.1-3.2\left(4 \mathrm{H}, \mathrm{m}, 2 \cdot \mathrm{NCH}_{2}\right), 3.0\left(2 \mathrm{H}, \mathrm{m}, \mathrm{NHCH}_{2}\right), 2.6-2.7$ $(1 \mathrm{H}, \mathrm{m}, \mathrm{COCH}), 2.3\left(2 \mathrm{H}, \mathrm{d}, \mathrm{NH}_{2}\right), 1.5-1.6\left(6 \mathrm{H}, \mathrm{m}, 3 \cdot \mathrm{CH}_{2}\right), 1.1-1.3$ $\left(6 \mathrm{H}, \mathrm{m}, \mathrm{CCH}_{2} \mathrm{C}\right)$. MS (FAB) $(\mathrm{m} / \mathrm{z}): 467(\mathrm{M}+1)$. Anal. calc. for $\mathrm{C}_{26} \mathrm{H}_{36} \mathrm{ClN}_{5} \mathrm{O}_{3}$ (\%): C 62.60, H 7.32, N 7.06; Found (\%): C 61.87, H 6.95, N 7.15.

$\mathrm{N}$-\{5-[2-(4-benzylpiperidin-1-ylmethyl)-1-oxoindan-5yloxy]-pentyl\}-6-hydrazinonicotinamide hydrochloride (33)

Yield $57 \%$. Mp. $177-180^{\circ} \mathrm{C}$. IR (KBr) $\mathrm{cm}^{-1}$ : 741, 1641, 1690, $2700,2985,3229 .{ }^{1} \mathrm{H}$ NMR (DMSO) $\delta: ~ 8.4$ (1H, s, Ar), $8.2(1 \mathrm{H}, \mathrm{s}$, CONH), 7.8 (1H, d, Ar), 7.7 (1H, s, Ar), 7.2-7.4 (5H, m, Ar), 6.8-6.9 $(2 \mathrm{H}, \mathrm{m}, \mathrm{Ar}), 6.5(1 \mathrm{H}, \mathrm{d}, \mathrm{Ar}), 4.1\left(2 \mathrm{H}, \mathrm{t}, \mathrm{OCH}_{2}\right), 3.6\left(2 \mathrm{H}, \mathrm{d}, \mathrm{CH}_{2} \mathrm{CH}\right)$, $3.4\left(2 \mathrm{H}, \mathrm{d}, \mathrm{NCH}_{2}\right), 3.3(1 \mathrm{H}, \mathrm{s}, \mathrm{CNH}), 3.1-3.2\left(4 \mathrm{H}, \mathrm{m}, 2 \cdot \mathrm{NCH}_{2}\right), 2.9$ $\left(2 \mathrm{H}, \mathrm{m}, \mathrm{NHCH}_{2}\right), 2.8\left(2 \mathrm{H}, \mathrm{t}, \mathrm{ArCH}_{2}\right), 2.4-2.5(1 \mathrm{H}, \mathrm{m}, \mathrm{COCH}), 2.1$ $\left(2 \mathrm{H}, \mathrm{d}, \mathrm{NH}_{2}\right), 1.3-1.7\left(5 \mathrm{H}, \mathrm{m}, 2 \cdot \mathrm{CH}_{2}, 1 \cdot \mathrm{CH}\right), 1.2-1.3(6 \mathrm{H}, \mathrm{m}$, $\left.\mathrm{CCH}_{2} \mathrm{C}\right)$. MS (FAB) (m/z): $558(\mathrm{M}+1)$. Anal. calc. for $\mathrm{C}_{33} \mathrm{H}_{42} \mathrm{ClN}_{5} \mathrm{O}_{3}$ (\%): C 66.93, H 7.15, N 11.83; Found (\%): C 66.68, H 6.96, N 11.69.

\section{Inhibition Studies on AChE and BChE}

$\nabla$

The activity of acetylcholinesterase (AChE) and butyrylcholinesterase inhibitors was measured spectrophotometricaly according to the colorimetric method of Ellman (1961) with some modification. The AChE activity was determined in a mixture containing the assay solution consisting of a phosphate buffer $(0.1 \mathrm{M}, \mathrm{pH} 8.0)$ with the addition of a solution of 5,5'-dithiobisnitrobenzoic acid (DTNB, $0.05 \mathrm{~mL}, 0.5 \mathrm{M}$ ), AChE $(5 \mathrm{U} / \mathrm{mL})$ and the appropriate inhibitor (tested compounds). The final assay volume was $3 \mathrm{~mL}$.

Inhibitor curves of the different derivatives were obtained using 7 concentrations of acetyltiocholine iodide as the substrate of the enzymatic reaction lasting $1 \mathrm{~min}$. Enzyme activity was determined by measuring the absorbance at $412 \mathrm{~nm}$ after $1 \mathrm{~min}$ at $37^{\circ} \mathrm{C}$ with Perkin Elmer Lambda 25 UV/VIS Spectrophotometer. Sample without inhibitor was consistently available to provide $100 \%$ of $\mathrm{AChE}$ activity. The reaction rates and the percent of inhibition due to the presence of tested compounds were compared. Each reaction was independently repeated at least 3 times.

Determination of butyrylcholinesterase (BChE) inhibitory activity was carried out in a similar manner using 5 units/L of $\mathrm{BChE}$ instead of AChE in the final volume of $3 \mathrm{~mL}$.

The $\mathrm{IC}_{50}$, defined as the concentration of each compound that reduces the enzymatic activity (AChE or BChE) by $50 \%$ with respect to that without inhibitors, was calculated by non-linear and linear regression.

DTNB, enzymes (C2629 and C4290) and acetylthiocholine iodide were purchased from Sigma-Aldrich.

\section{Results and Discussion}

\section{$\nabla$}

\section{Chemistry}

In the present investigation compound $\mathbf{2}$ was synthesized following the method described by Abrams ( $\bullet$ Fig. 1) [12]. 6-chloronicotinic acid and hydrazine hydrate were refluxed at $100^{\circ} \mathrm{C}$. In the next step, hydrazine $\mathrm{N}$-atom was protected by di-tert butyl dicarbonate $\left((\mathrm{t}-\mathrm{BuOCO})_{2} \mathrm{O}\right)$ to give 6-Boc-hydrazinopyridine-3-carboxylic acid 2. Condensation commercially available 5-hydroxyindan-1-on and secondary amines (piperidine 3, 4-benzylpiperidine 4) with paraformaldehyd gave 5, 6 ( 0 Fig. 2) $[13,14]$. In the next step, derivatives of 5-hydroxyindan-1-one $\mathbf{5}$, 6 coupled with alkyl linker through etheric bond. Based on our study, we determined 2 methods for synthesizing compounds with a variable length of the carbon chain at C-5. N-Boc-aminoethoxy derivatives $\mathbf{1 0}, \mathbf{1 1}$ were used to perform O-alkylation of $\mathbf{5 , 6}$ with N-Boc-2-bromoethylamine $\mathbf{7}$ using potassium carbonate in refluxing acetonitrile [15]. Similar reaction conditions employing N-Boc-3-bromopropylamine $\mathbf{8}$, led to the desired $\mathbf{1 2}$, 13 ( $\odot$ Fig. 3a). Treatment of 5, 6 under Mitsunobu conditions, using 5 -aminopentanol with $\mathrm{N}$-atom protected by Boc $\mathbf{9}$ provided 14, 15 ( $\odot$ Fig. 3b) [16-18]. N-atom of n-bromoalkylamine and $\mathrm{N}$-atom of 5-aminopentanol were protected following the methods described previously $[15,19]$. All the obtained N-Boc-aminoalkoxy derivatives $\mathbf{1 0 - 1 5}$ were deprotected in the presence of ether saturated $\mathrm{HCl}$, affording 16-21 ( $\bullet$ Fig. 3c). Coupling reac- 

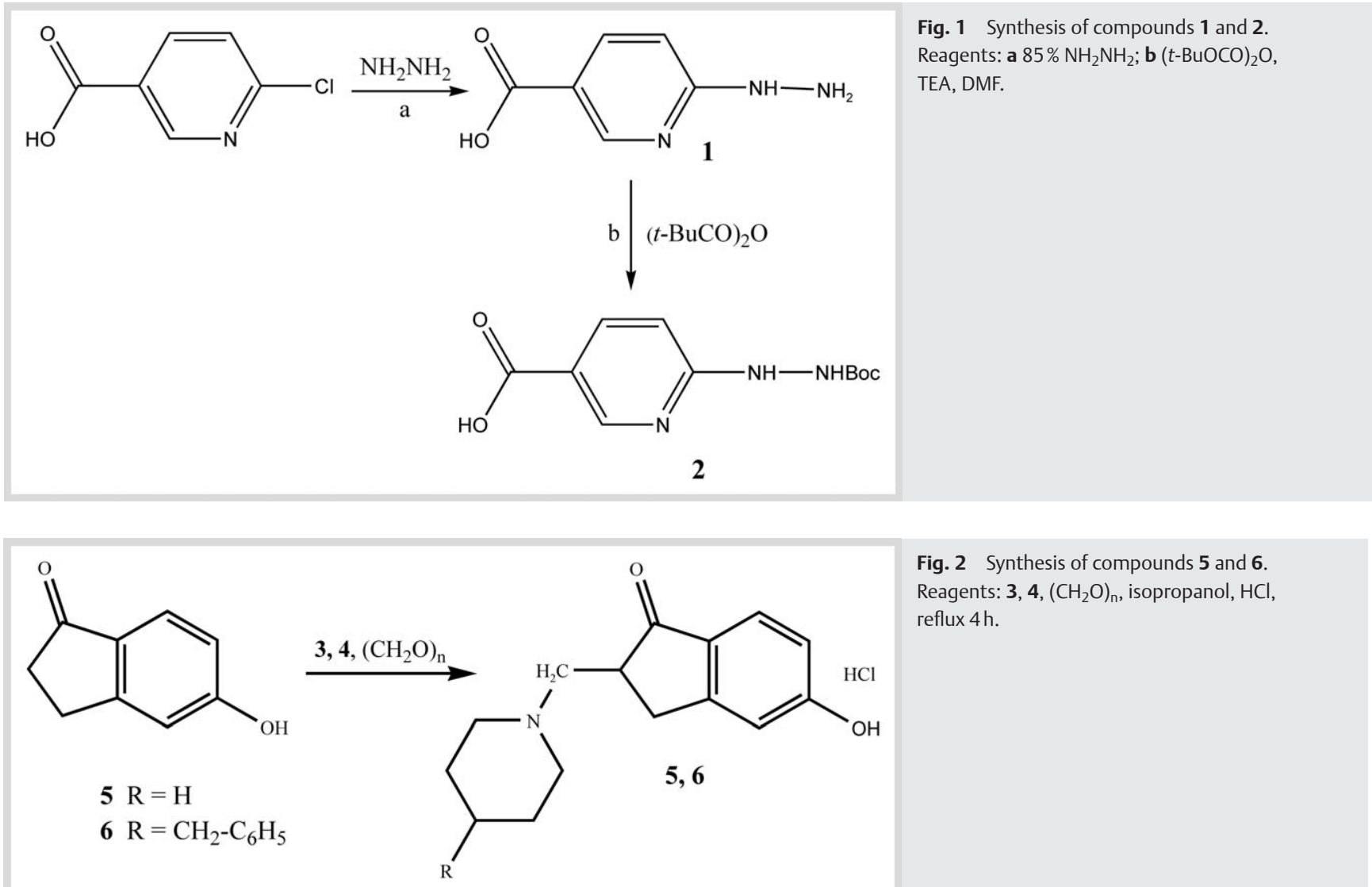

Fig. 2 Synthesis of compounds $\mathbf{5}$ and $\mathbf{6}$. Reagents: 3, 4, $\left(\mathrm{CH}_{2} \mathrm{O}\right)_{n}$, isopropanol, $\mathrm{HCl}$, reflux $4 \mathrm{~h}$.

$$
\begin{aligned}
& 5 \mathrm{R}=\mathrm{H} \\
& 6 \mathrm{R}=\mathrm{CH}_{2}-\mathrm{C}_{6} \mathrm{H}_{5}
\end{aligned}
$$

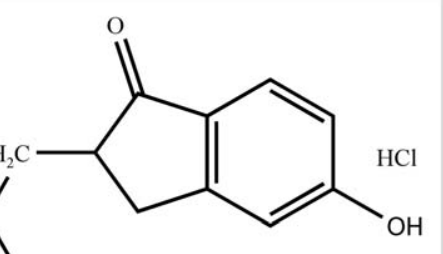

5,6

tion of the intermediates 16-21 with 6-Boc-hydrazinopyridinecarboxylic acid $\mathbf{2}$ in the presence carbonyldiimidazole (CDI) led to the formation of the desired compound 22-27 ( Fig. 3d) [20-22]. The final compounds 28-33 were obtained from 22-27, which were deprotected in the presence of the ether saturated $\mathrm{HCl}(\odot$ Fig. 3e).

\section{Biochemistry}

Anticholinesterase activities of the obtained compounds 28-33 were determined by modification of Ellman's spectrophotometric method.

Parameters of the enzymatic reaction $\mathrm{K}_{\mathrm{m}}$ and $\mathrm{V}_{\text {max }}$ were obtained by linear regression of the reaction rate as a function of substrate concentration ( $\bullet$ Table 1). To determine the type of inhibition, the Michaelis-Menten equation was plotted using LineweaverBurk linear transformation (1/v vs. $1 /[S]) . K_{i}$ constans were then calculated using non linear regresion [23-26].

The inhibitory activities against both $\mathrm{AChE}$ and $\mathrm{BChE}$ of the compounds 28-33 together with the reference donepezil are reported in - Table 2 , expressed as $\mathrm{IC}_{50}$ values [27]. All the tested compounds showed a higher inhibitory activity on AChE than BChE, and showed lower inhibition of AChE compared with donepezil. Compound $\mathbf{3 3}$ showed the greatest affinity for AChE, while compound $\mathbf{2 8}$ was found to be the least effective against this enzyme.

We also assessed the impact of the activity of the constructed derivatives obtained against AChE. After analyzing biological activity of the compounds with the same length of carbon chain, it can be concluded that replacing piperidine with benzylpiperidine substituent in compounds 28, 30, and 32 (and obtaining compounds 29,31 , and 33 , respectively) was reflected by a slight increase in activity against AChE. For example, the activity of ethyl derivative of benzylpiperidine $\mathbf{2 9}$ increased 9-fold as compared to the ethyl derivative of piperidine 28. Variation of the alkyl linker length between the 2 groups, indanone and Hynic, for piperidine derivatives $\mathbf{2 8 ,} \mathbf{3 0}, \mathbf{3 2}$ revealed the penthylenelinked substance to be more active than their propylene and ethylene linked counterparts. In the case of derivatives of $\mathrm{N}$-benzylpiperidine $\mathbf{2 9}, \mathbf{3 1}, \mathbf{3 3}$, the influence of alkylene-linker length was negligible and compounds $\mathbf{3 1}$ and $\mathbf{3 3}$ showed nearly equal potency in anti-AChE assay.

All the tested compounds showed higher inhibition of BChE than the reference (donepezil).

The selectivity toward $\mathrm{AChE}$ ( $\mathrm{IC}_{50}$ ratio of $\mathrm{BChE} / \mathrm{AChE}$ ) and $\mathrm{BChE}$ ( $\mathrm{IC}_{50}$ ratio of $\mathrm{AChE} / \mathrm{BChE}$ ) of the obtained compounds and reference inhibitor (donepezil) were also determined. All the tested compounds showed a higher selectivity for AChE than for BChE, while the selectivity with respect to AChE was less than in the case of the standard (donepezil), selectivity to BChE was greater than for donepezil. Compound $\mathbf{3} 3$ was found to be the most potent with regards to AChE activity and the most selective for AChE among the obtained indanone derivatives. The selectivity of this derivative was nearly 50-fold higher compared to the least selective derivative 28. Concurrently, compound $\mathbf{2 8}$ was characterized by the highest selectivity against BChE.

\section{Drug-modeling studies}

Docking studies of donepezil-hydrazinonicotinamide hybrids on the active site of electric eel AChE inhibited by donepezil (PDB: 1EA5) revelated their mode of structural and positional requirements for potential activity. These studies elucidated the interaction between electric eel AChE and inhibitors 29-33. The AChE-Inhibitor complex was generated using Cache software (Fujitsu). Docking analysis performed with these inhibitors 


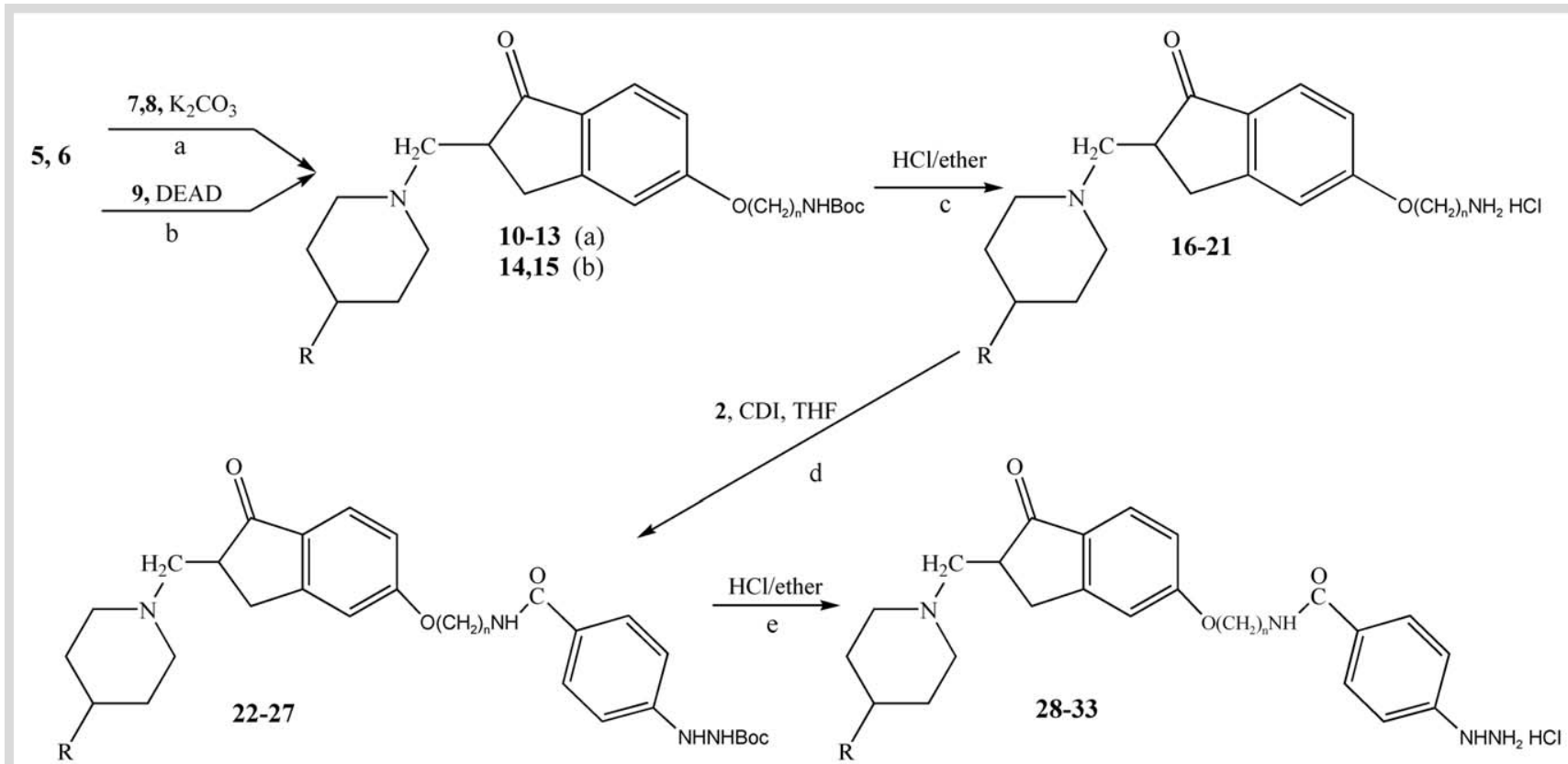

13, 19, 25, $31 \quad n=3, R=\mathrm{CH}_{2}-\mathrm{C}_{6} \mathrm{H}_{5}$

14, 20, 26, $32 \mathrm{n}=5, \mathrm{R}=\mathrm{H}$

11, 17, 23, $29 \quad \mathrm{n}=2, \mathrm{R}=\mathrm{CH}_{2}-\mathrm{C}_{6} \mathrm{H}_{5}$

$15,21,27,33 n=5, R=\mathrm{CH}_{2}-\mathrm{C}_{6} \mathrm{H}_{5}$

Fig. 3 Synthesis of compounds 10-33: a 7, 8, $\mathrm{K}_{2} \mathrm{CO}_{3}$, acetonitryl, reflux, $12 \mathrm{~h}$; b 9, Ph ${ }_{3} \mathrm{P}, \mathrm{DEAD}$, TEA, THF; $\mathbf{c} \mathrm{HCl} / \mathrm{ether;} \mathbf{d} \mathrm{CDI}, \mathrm{TEA}$, THF, room temp., 20 h; e $\mathrm{HCl} /$ ether.

Table 1 Statistical parameters and values of $\mathrm{K}_{\mathrm{m}}$ and $\mathrm{V}_{\max }$ for $\mathrm{AChE}$ and BChE.

\begin{tabular}{lll} 
Parameters & AChE & BChE \\
$\mathrm{K}_{\mathrm{m}}(\mu \mathrm{M})$ & 0.096453 & 0.082586 \\
$\mathrm{~V}_{\max }(\mu \mathrm{M} / \mathrm{mL} / \mathrm{min})$ & 0.103247 & 0.094610 \\
\hline $\mathrm{R}^{2}$ & 0.9895 & 0.9857 \\
\hline Standard error & 0.0193 & 0.0017 \\
\hline
\end{tabular}

Table $2 \quad I_{50}$ values for activities on $\mathrm{AChE}$ and $\mathrm{BChE}$.

\begin{tabular}{|c|c|c|c|c|}
\hline $\begin{array}{l}\text { Com- } \\
\text { pounds }\end{array}$ & $\begin{array}{l}\text { AChE inhibi- } \\
\text { tion }\left(\mathrm{IC}_{50}, \mu \mathrm{M}\right)\end{array}$ & $\begin{array}{l}\text { BChE inhibi- } \\
\text { tion }\left(\mathrm{IC}_{50}, \mu \mathrm{M}\right)\end{array}$ & $\begin{array}{l}\text { Selectivity } \\
\text { for } \mathrm{AChE}^{\dagger}\end{array}$ & $\begin{array}{l}\text { Selectivity } \\
\text { for BChE }\end{array}$ \\
\hline 28 & $1.879 \times 10^{-4}$ & $1.026 \times 10^{-3}$ & 5.459 & 0.183 \\
\hline 29 & $2.066 \times 10^{-5}$ & $1.285 \times 10^{-3}$ & 62.204 & 0.017 \\
\hline 30 & $1.164 \times 10^{-4}$ & $8.981 \times 10^{-4}$ & 7.715 & 0.130 \\
\hline 31 & $1.768 \times 10^{-5}$ & $2.233 \times 10^{-3}$ & 126.464 & 0.008 \\
\hline 32 & $4.229 \times 10^{-5}$ & $1.431 \times 10^{-3}$ & 33.817 & 0.029 \\
\hline 33 & $1.087 \times 10^{-5}$ & $2.659 \times 10^{-3}$ & 248.776 & 0.004 \\
\hline donepezil & $0.601 \times 10^{-5}$ & $3.444 \times 10^{-3}$ & 573.096 & 0.002 \\
\hline
\end{tabular}

† Selectivity for $\mathrm{AChE}$ is defined as $\mathrm{IC}_{50}(\mathrm{BChE}) / \mathrm{IC}_{50}(\mathrm{AChE})$

$\ddagger$ Selectivity for $B C h E$ is defined us $I C_{50}(A C h E) / I C_{50}(B C h E)$

show that compounds 29-33 bind with the total score of -184 . 794, -196.681, $-165.113,-195.338,-181,939$, respectively. (० Fig. 4)

\section{Conclusion}

\section{$\nabla$}

In summary, series of indanone derivatives with hydrazine nicotinate moiety were synthesized and their anticholinesterase

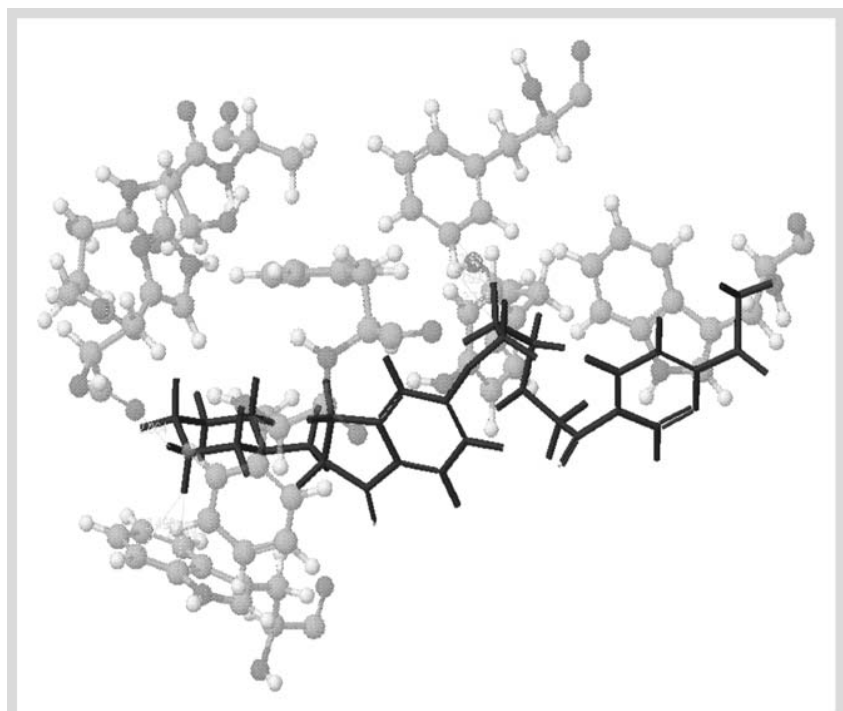

Fig. 4 The model of docking of compound 33 with main aminoacids of AChE active site.

activities were evaluated. All tested compounds exhibited a higher affinity to AChE than for BChE. Similarly, their selectivity for $\mathrm{AChE}$ was greater than for BChE. When compared to donepezil, all synthesized molecules were less active with regards to inhibiting $\mathrm{AChE}$ and slightly more active in inhibiting $\mathrm{BChE}$. All tested derivatives were less selective for AChE and BChE, their selectivity is higher than the reference (donepezil). The results indicate that compound $\mathbf{3 3}$ (N-benzylpiperidine derivative) was the most effective inhibitor of AChE, additionally, it 
had the highest selectivity for the enzyme. Compound $\mathbf{3 0}$ (piperidine derivative) was most active against $\mathrm{BChE}$, however, compound $\mathbf{2 8}$ showed the highest selectivity for $\mathrm{BChE}$.

\section{Acknowledgments}

This study is supported by the Medical University of Lodz, Poland (grant No 502-13-336).

\section{Conflict of Interest}

The authors have declared no conflict of interest.

\section{References}

1 Leifer PB. Early diagnosis of Alzheimer's disease: clinical and economic benefits. J Am Geriatr Soc 2003; 51: S281-S288

2 Barrio JR, Satyamurthy N, Huang $S$ et al. Dissecting molecular mechanisms in the living brain of dementia patients. Acc Chem Res 2009; 42: 842-850

3 Jagust $W$. Molecular neuroimaging in Alzheimer's disease. NeuroRx 2004; 1: 206-212

4 Blennow K, de Leon MJ, Zetterberg $H$. Alzheimer's Disease. Lancet 2006; 368: 387-403

5 Cokugras A. Butyrylcholinesterase: structure and physiological importance. Turk J Biochem 2003; 85: 54-61

6 Ellis JM. Cholinoesterase inhibitors in the treatment of dementia. J Am Osteopath Assoc 2005; 105: 145-148

7 Camps $P$, Munoz-Torrero $D$. Cholinergic drugs in pharmacotherapy of Alzheimer's disease. Mini Rev Med Chem 2002; 2: 11-25

8 Calabria M, Geroldi C, Lussignoli G et al. Efficacy of acetyl-cholinestraseinhibitor (ACHEI) treatment in Alzcheimer's disease: A 21-month follow-up real world study. Archives of Gerontology and Geriatrics 2009; 49: e6-e11

9 Sugimoto $H$. The new approach in development of anti-Alzheimer's disease drugs via the cholinergic hypothesis. Chem Biol Interact 2008; 175: 204-208

10 Musiat A, Bajda M, Malawska B. Recent developments in cholinesterases inhibitors for Alzheimer's disease treatment. Curr Med Chem 2007; 14: 2654-2679

11 Ellman GL, Courtney KD, Andres $V$ et al. A new rapid colorimetric determination of acetylcholinesterase activity. Biochem Pharmacol 1961; 7: 88-95

12 Abrams MJ. Technetium-99m-human polyclonal IgG radiolabeled via the hydrazine nicotinamide derivative for imaging focal sites of infection in rats. J Nucl Med 1990; 31: 2022-2028

13 Lorand T, Kocis B, Sohar $P$ et al. Synthesis and antibacterial study of unsaturated Mannich ketones. Eur J Med Chem 2001; 36: 705-717
14 Comanita E, Roman G, Popovici I et al. Synthesis and reactivity of some Mannich bases. VIII. Studies on several Mannich bases derived from ortho- hydroxylacetophenones and their conversion into oximino derivatives. J Serb Chem Soc 2001; 66: 2-16

15 Um S, Park SH, Park CH et al. Synthesis of novel quinolinecarboxamide derivatives with estrogenic activity. Bull Korean Chem Soc 2003; 24: 677-680

16 Mitsunobu 0 . The use of diethyl azodicarboxylate and triphenylphosphine in synthesis and transformation of natural products. Synthesis 1981; 1 : 1-84

17 Marugan JJ, Manthey C, Anaclerio B et al. Design, synthesis and biological evaluation of novel evaluation of novel potent and selective $\alpha_{v} \beta_{3} /$ $\alpha_{v} \beta_{5}$ integrin dual inhibitor with improved bioavailability. Selection of the molecular core. J Med Chem 2005; 48: 926-934

18 Hinshaw JC, Suh DJ, Garnier P. Oxidosqualene Cyclase Inhibitors as Antimicrobal Agents. J Med Chem 2003; 46: 4240-4243

19 Lee BH, Miller MJ. Natural ferric ionophores: total synthesis of schizokinen, schizokinen A and arthrobactin. J Org Chem 1983; 48: 24-31

20 Paul R, Anderson GW. N,N'-Carbonyldiimidazole, a new peptide forming reagent. J Am Chem Soc 1960; 82: 4596-4600

21 Vaidynathan R, Kalthod VG, Manley JM et al. Amidations using N,N'carbonyl diimidazole: remarkable rate enhancement by carbon dioxide. J Org Chem 2004; 69: 2565-2568

22 Alonso D, Dorronsoro I, Rubio L et al. Donepezil - tacrine hybrid related derivatives as new dual binding site inhibitors of AChE. Bioorg Med Chem 2005; 13: 6588-6597

23 Shan WJ, Huang L, Zhou $Q$ et al. Synthesis, biological evaluation of 9-N-substituted berberine derivatives as multi-functional agents of antioxidant, inhibitors of acetylcholinesterase, butyrylcholinesterase and amyloid aggregation. Eur J Med Chem 2011; 46: 5885-5893

24 Szymański P, Żurek E, Mikiciuk-Olasik E. New tacrine-hydrazinonicotinamide hybrids as acetylcholinesterase inhibitors of potential interest for the early diagnostics of Alzheimer's disease. Pharmazie 2006; 61: 269-273

25 Szymański P, Janik A, Żurek E et al. Design, synthesis and biological evaluation of new 2-benzoxazoline derivatives as potential cholinesterase inhibitors for therapy of Alzheimer's disease. Pharmazie 2011; 66: 399-403

26 Szymański P, Markowicz M, Mikiciuk-Olasik E. Synthesis and biological activity of derivatives of tetrahydroacridine as acetylcholinesterase inhibitors. Bioorganic Chemistry 2011; 39: 138-142

27 Cheng YC, Prusoff WH. Relationship between the inhibition constants $\left(\mathrm{K}_{\mathrm{i}}\right)$ and the concentration of the inhibitor which causes 50 percent inhibition $\left(\mathrm{IC}_{50}\right)$ of an enzymatic reaction. Biochem Pharmacol 1973; 22: 3099-3108 